\title{
A PRELIMINARY STUDY OF SEXUAL DIMORPHISM OF SCAPULA BY COMPUTED TOMOGRAPHY IN THE MALAYSIAN POPULATION
}

\author{
NORMALIZA OMAR ${ }^{1,2}$, SITI HANUM MOHD ALI ${ }^{1,2}$, MOHAMED SWARHIB SHAFIE ${ }^{1}$, NIK AZUAN NIK ISMAIL ${ }^{3}$, \\ HELMI HADI $^{4}$, FARIDAH MOHD NOR ${ }^{1 *}$
}

${ }^{1}$ Forensic Unit, Department of Pathology, Universiti Kebangsaan Malaysia Medical Centre, Jalan Yaacob Latiff, Bandar Tun Razak, 56000 Kuala Lumpur, Malaysia. ${ }^{2}$ Department of Basic Medical Sciences, Faculty of Medicine and Health Sciences, Universiti Sains Islam Malaysia, Menara B, Persiaran MPAJ, Jalan Pandan Utama, 56100 Pandan Indah, Kuala Lumpur, Malaysia. ${ }^{3}$ Department of Radiology, Universiti Kebangsaan Malaysia Medical Centre, Jalan Yaacob Latiff, Bandar Tun Razak, 56000 Kuala Lumpur, Malaysia. ${ }^{4}$ Forensic Unit, School of Health Sciences, Universiti Sains Malaysia, 16150 Kubang Kerian, Kelantan, Malaysia. Email: mnfaridah@gmail.com

Received: 10 October 2018, Revised and Accepted: 29 November 2018

ABSTRACT

Objective: Sex estimation is one of the crucial steps for human identification, which is evident in cases of commingled, eroded, and/or missing remains. When pelvis or skull are unavailable, scapula has been used as an alternative bone for determining sex. Besides, the scapula was shown to be population-specific in several studies. Limited dry bone collections in Malaysia have led to various recommendations of virtual anthropology studies of bone in human identification. The aims of this study were to investigate the sexual dimorphism of the scapula using three-dimensional (3D) computed tomography (CT) imaging and to generate population-specific equations for sex determination in the Malaysian population.

Methods: A total of $66 \mathrm{CT}$ thorax images of 33 males and 33 females were taken. Morphological breadth (MB) and morphological length (ML) on bilateral scapulae were measured on 3D CT reconstructed images. Independent t-test and discriminant function analysis (DFA) were performed for analysis.

Results: Results revealed that both parameters showed sexual dimorphism of scapula but displayed no difference between the right and left scapulae. DFA showed that MB and ML had high accuracy for sex estimation. The equations were highly accurate when both parameters were used in combination, followed by MB only and ML only, in that sequence.

Conclusion: In brief, scapula measurements may be useful for forensic assessment of sex in the Malaysian population.

Keywords: Forensic, Morphological, Scapula, Sex estimation, Three-dimensional computed tomography.

(c) 2019 The Authors. Published by Innovare Academic Sciences Pvt Ltd. This is an open access article under the CC BY license (http://creativecommons. org/licenses/by/4. 0/) DOI: http://dx.doi.org/10.22159/ajpcr.2019.v12i1.30233

\section{INTRODUCTION}

Forensic identification of human remains is one of the most challenging parts in the forensic investigation. In cases of severe mutilation and advanced decomposition in human remains, biological profiles such as sex, age, stature, and ethnic must be determined to allow a positive identification. Accurate estimation of sex is clearly an important consideration toward facilitating the identification of an unknown individual because estimation of age at death and stature is sexdependent [1]. Besides, it reduces the possible matches by half by identifying the sex of human remains [2].

Identification of skeletonized remains by forensic anthropologist is usually done by either the morphological or metric methods. The morphological method requires vast experience, where visual assessment is made on dry bones, which depends much on the subjectivity of the observer. Another method is the metric analysis, where predictions are made based on equations or models from statistical methods [3]. Metric analysis usually derives from bone measurements or radiological bone images. Alternatively, identification can be done by combining both methods, which may be useful in cases, where the remaining bone is indefinite and altered in architecture and morphology. In cases of rheumatoid arthritis, certain degree of inflammation may predict bone loss, which might be of value in identifying the risk of developing osteoporosis [4]. This can lead to a fracture that will affect the identification of human remains.

Sex estimation has been widely done on pelvis and cranium, as they provide the most accurate assessment of sex [5]. If these bones were not available or severely crushed, other bones may be used for identification purposes. Several studies have been using long bones [6,7], metatarsal [8], and vertebral bodies [9] as alternatives for sex estimation. Nevertheless, the scapula is also one of the bones that manifest sexually dimorphic features $[10,11]$. The reason being, that after growth response has stopped, the changes in scapula are almost insignificant in its sexuality [11].

Several studies have been conducted on the estimation of sex from scapula [10-15], where various human groups displayed different skeletal features depending on their geographical areas and genetic pattern $[5,16]$. These studies had used either dry skeletal collection [12-14] or multi-slice computed tomography (CT) for sex estimation $[10,11,15,17]$. Over the recent decades, multi-slice CT has been extensively used in forensic anthropology. It allows researchers to deal with issues using traditional anthropological approaches, without compromising the bony remains [18].

In Malaysia, there is a paucity of studies on bone morphometry focusing on identification of skeletonized remains. Limited skeletal collection and sources of biological data are the main challenges for these restrictions. With the increased trend of mass disaster in Malaysia such as massive flood and landslide, along with the Malaysia airline plane crash in Ukraine, it leads to demanding needs of identification of human remains based on the Malaysian population. With such limitation, there were only a few studies in Malaysia using CT scan of crania and mandible for forensic identification [19-21]. Besides, there is no such study using scapula for identification in Malaysia. For these reasons, this study was 
conducted to establish criteria for sex estimation in scapula by threedimensional (3D) CT and to generate population-specific equations for estimating sex in the Malaysian population.

\section{METHODS}

\section{Subjects}

The study was conducted retrospectively using CT thoraximages retrieved from the Radiology Department, Universiti Kebangsaan Malaysia Medical Center from 2015 to 2017. The ethics approval was granted by the University Ethics Committee (Ethic no: UKM PPI/111/8/JEP-2018004). The data comprised 66 subjects with 33 males and 33 females, aged between 25 and 60 years (mean age: 40 years). Only subjects with intact scapula by CT scan were included in this study, while subjects with scapula fractures, congenital anomalies, and/or back injuries, that might affect scapula dimensions, were excluded from the study. Apart from that, subjects aged $>60$ were excluded due to increased incidence of osteoporosis [22]. The CT was performed by Siemens SOMATOM Sensation 64 (Siemens Germany Ltd.) with $0.5 \mathrm{~mm}$ slices thickness, and auto-programmed for reconstruction at $1.0 \mathrm{~mm}$ for better resolution. The 3D-reconstructed images were done by volume rendering technique of the CT images. Osiri $X^{\circledR}$ software was used to segment the 3D images of the CT scan so that the whole scapula could be visualized.

\section{Data collection}

All specimens were anonymous and blinded to the observers during the measurements. The measurements were taken to the nearest $0.01 \mathrm{~cm}$ $(0.1 \mathrm{~mm})$ according to the method by Zhang et al. (2016). The measured parameters were as follows:

1. Morphological breadth (MB) - the distance between the medial margin and middle of the margin of the glenoid cavity (Fig. 1).

2. Morphological length (ML) - the distance between the end of the inferior angle and vertex of the superior angle (Fig. 2).

\section{Error measurements}

For intraobserver and interobserver variability, 20 randomly selected CT images were re-measured from the reconstructed 3D images of both scapulae. They were re-measured by the co-researcher and first researcher. The intraobserver and interobserver variability was performed by calculating the relative technical error of measurements (rTEM) and coefficient reliability (R) of the data. Intraobserver variability refers to the consistency of each measurement in the same observer on the same sample at different times. By contrast, interobserver variability refers to the consistency of each measurement between two observers on the same sample [23].

\section{Statistical analysis}

Statistical analyzes were performed using SPSS version 21.0 (Microsoft Office 2013, Microsoft. Redmond, WA, USA). Independent $t$-test was used to analyze for any difference in measurements between the right and left scapula, and to analyze for any difference in measurements between sexes. Discriminant function analysis (DFA) was performed to generate equations for sex estimation. Wilk's lambda was yielded to determine the significance of the discriminant functions in male and female groups. The scale ranged from 0 to 1 , in which 0 means total discrimination and 1 means no discrimination. Leave-one-out cross-validation was used to determine the prediction accuracy rates in male and female groups [17].

\section{RESULTS}

The descriptive statistics (maximum, minimum, and mean and standard deviation) of bilateral scapulae were presented (Table 1). By independent $t$-test, there was no significant difference between the right and left scapula $(p<0.05)$. Hence, either scapula can be used for estimating sex. In this study, the left scapula was chosen for estimating sex.

The descriptive statistics of the left scapula in male and female were presented (Table 2), in which the mean values for MB and ML were significantly higher in males than in females. By independent t-test, there were significant differences for MB and ML between male and

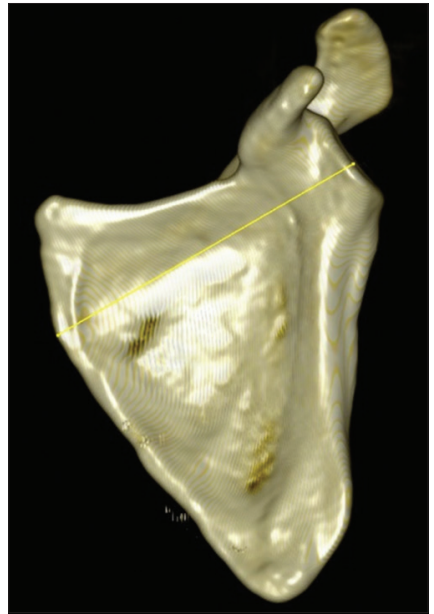

Fig. 1: Scapula in anterior view showing the morphological breadth (as marked by the line)

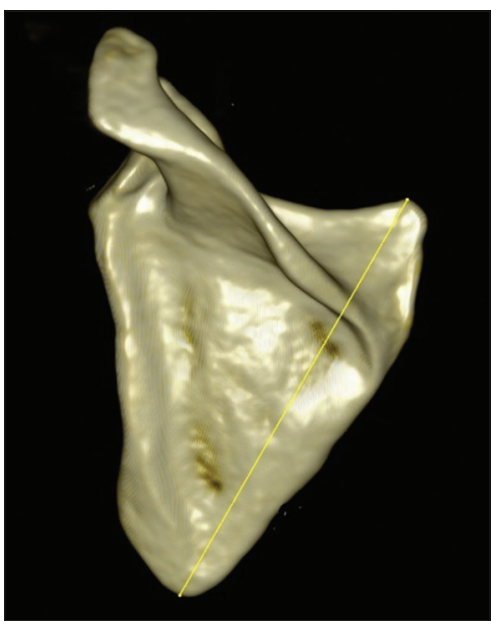

Fig. 2: Scapula in posterior view showing the morphological length (as marked by the line)

female $\left({ }^{*} \mathrm{p}<0.05\right)$. This indicated that sexual dimorphism was evident in scapula based on both parameters.

Univariate discriminant function and multivariate DFAs of the left scapula were presented (Table 3). By using MB only, the accuracy rate of the equations for male and female was $93.9 \%$ and $87.9 \%$, respectively. The overall accuracy rate of the equations was higher, when a combination of parameters was used, followed by MB only and ML only, in that sequence (Table 4).

The discriminant equation developed using MB only, was as follows:

Discriminant Scores $(D S)=(2.24 \times \mathrm{MB})-21.68$

The sectioning point is 0 so that if DS is more than 0 , the individual is male, and if DS is $<0$, the individual is female. Similarly, by using ML only, the DFA showed $84.8 \%$ accuracy in both male and female. The overall accuracy in determining sex was $84.8 \%$.

The discriminant equation developed using ML only, was as follows:

$D S=(1.13 \times \mathrm{ML})-19.62$

The sectioning point is 0 so that if DS $>0$, the individual is male, and if DS is $<0$, the individual is female. When both MB and ML were used in estimating sex, the overall accuracy rate was greater (92.4\%). 
Table 1: Independent t-test for MB and ML in the left and right scapula

\begin{tabular}{|c|c|c|c|c|c|c|c|c|c|}
\hline \multirow[t]{2}{*}{ Parameter } & \multicolumn{4}{|c|}{ Right scapula } & \multicolumn{4}{|c|}{ Left scapula } & \multirow[t]{2}{*}{$\mathbf{p}$} \\
\hline & $\mathbf{n}$ & Min. & Max. & Mean \pm SD & $\mathbf{n}$ & Min. & Max. & Mean \pm SD & \\
\hline MB & 66 & 8.24 & 11.10 & $9.57 \pm 0.66$ & 66 & 8.33 & 10.94 & $9.69 \pm 0.67$ & 0.31 \\
\hline
\end{tabular}

*MB: Morphological breadth, ML: Morphological length, n: Sample size, Min: Minimum, Max: Maximum. *p value was significant when P<0.05. SD: Standard deviation

Table 2: Independent t-test for MB and ML in male and female

\begin{tabular}{|c|c|c|c|c|c|c|c|c|c|}
\hline \multirow[t]{2}{*}{ Parameter } & \multicolumn{4}{|c|}{ Male } & \multicolumn{4}{|c|}{ Female } & \multirow[t]{2}{*}{$\mathbf{p}$} \\
\hline & $\mathbf{n}$ & Min & Max & Mean \pm SD & $\mathbf{n}$ & Min & Max & Mean \pm SD & \\
\hline MB & 33 & 9.30 & 10.94 & $10.20 \pm 0.45$ & 33 & 8.33 & 9.96 & $9.19 \pm 0.45$ & 0.001 \\
\hline
\end{tabular}

*MB: Morphological breadth, ML: Morphological length, n: Sample size, Min: Minimum, Max: Maximum. *p value was significant when P<0.05. SD: Standard deviation

Table 3: Univariate and multivariate DFA for MB and ML in male and female

\begin{tabular}{|c|c|c|c|c|c|c|}
\hline \multirow{2}{*}{$\begin{array}{l}\text { Parameter } \\
\text { coefficients }\end{array}$} & \multirow[t]{2}{*}{$\mathbf{n}$} & \multirow[t]{2}{*}{ Unstandardized coefficients } & \multicolumn{2}{|c|}{ Group centroid } & \multirow[t]{2}{*}{ Sectioning points } & \multirow[t]{2}{*}{ Wilk's Lambda } \\
\hline & & & Male & Female & & \\
\hline MB only & 66 & 2.24 & 1.13 & -1.13 & 0 & 0.43 \\
\hline Constant & & -21.68 & & & & \\
\hline ML only & 66 & 1.13 & 1.19 & -1.19 & 0 & 0.41 \\
\hline Constant & & -19.62 & & & & \\
\hline MB & 66 & 1.32 & 1.44 & -1.44 & 0 & 0.32 \\
\hline Constant & & -25.52 & & & & \\
\hline
\end{tabular}

*MB: Morphological breadth, ML: Morphological length, n: Sample size, DFA: Discriminant function analysis

The discriminant equation for sex estimation using both parameters was as follows:

$D S=\{(1.32 \times \mathrm{MB})+(0.93 \times \mathrm{ML})\}-25.52$

The sectioning point is 0 so that if DS $>0$, the individual is male, and if DS is $<0$, the individual is female. A Wilk's lambda test was performed to analyze how well each independent parameter contributes to the model. The scales range from 0 to 1 , that is, 0 means total discrimination and 1 means no discrimination. It means that the closer Wilk's lambda is to 0 , the more the parameter contributes to the discriminant function.

The interobserver and intraobserver variability was performed by two observers for 20 samples (Table 5). The acceptable ranges for rTEM for a beginner anthropometrist were $<1.5 \%$ for intraobserver and were $<2.0 \%$ for interobserver [24]. In the present study, both interobserver and intraobserver variability showed rTEMs of $<1.5 \%(0.61-0.71 \%)$ and $<2.0 \%(0.82-0.98 \%)$, respectively. The coefficient reliability, $R$ values for both parameters were $>0.75(0.97-0.99)$.

\section{DISCUSSION}

The present study had focused on sex estimation from reconstructed 3D CT of the scapula. To the best of our knowledge, this is the first study conducted in Malaysia that used 3D scapula for sex estimation. It has provided baseline data of scapula measurements and generated population-specific equations for the Malaysians.

In several studies on bilateral symmetry of the scapula, it was exhibited that there was no significant difference between the right and left scapula [10-12,14]. However, Dabbs and Moore-Jansen (2010) and Hudson et al. (2016) indicated the presence of bilateral asymmetry in the right and left scapula $[25,26]$. In the present study, there was bilateral symmetry of the scapula, which means that in cases, where scapula is discovered, whether right or left side, it can either be used for sex estimation using the present equations.
Table 4: Correct prediction rates for equations in original sample and cross-validated samples

\begin{tabular}{llllllll}
\hline \multirow{2}{*}{ Parameters } & \multicolumn{2}{l}{ Correctly classified (\%) } & & \multicolumn{2}{l}{ Cross validated (\%) } \\
\cline { 2 - 4 } \cline { 7 - 8 } & Male & Female & Overall & & Male & Female & Overall \\
\hline MB only & 93.9 & 87.9 & 90.9 & & 93.9 & 87.9 & 90.9 \\
ML only & 84.8 & 84.8 & 84.8 & & 84.8 & 84.8 & 84.8 \\
Both MB and ML & 87.9 & 97.0 & 92.4 & & 87.9 & 97.0 & 92.4 \\
\hline
\end{tabular}

*MB: Morphological breadth, ML: Morphological length

Table 5: Interobserver and intraobserver variability in two observers in the measurements of scapula

\begin{tabular}{lllllll}
\hline \multirow{2}{*}{ Parameters } & $\mathbf{n}$ & \multicolumn{2}{c}{ Intraobserver } & & \multicolumn{2}{c}{ Interobserver } \\
\cline { 3 - 4 } \cline { 7 - 7 } & & rTEM, \% & $\mathbf{R}$ & & rTEM, \% & $\mathbf{R}$ \\
\hline MB & 20 & 0.71 & 0.99 & & 0.82 & 0.99 \\
ML & 20 & 0.61 & 0.99 & & 0.98 & 0.97 \\
\hline
\end{tabular}

*MB: Morphological breadth, ML: Morphological length, rTEM: Relative technical error of measurements, R: Coefficient reliability

The scapula was shown to be sexually dimorphic in several researches. In the Greek population, Papaioannou et al. (2012) explained a good separation ( $93 \%$ of total variability) between different sexes from scapula measurements using principal component analysis. Koukiasa et al. (2017) suggested that scapula was sexually dimorphic, where male scapula was larger than female in many parameters [27]. Metric analysis of the scapula by Dabbs and Moore-Jansen (2010), who measured 23 parameters, also showed that the parameters were sexually dimorphic. Studies in Asian countries such as the Chinese population [17], Japanese [10], and Thai [14] populations had documented significant differences between male and female scapula. Both Zhang et al. (2016) and Torimitsu et al. (2016) were using reconstructed 3D images in their studies. In the present work, the mean values for both MB and ML yielded that male scapula was significantly larger than female $(\mathrm{p}<0.01)$. These results proved that scapula is useful as a sex indicator for the Malaysians. 
Further analyzes were conducted to generate discriminant equations from the parameters, MB, and ML. The univariate and multivariate discriminant functions were developed with sectioning points by cross-validation analysis. For univariate discriminant analysis, the equations can be used in cases, where only one measurement can be obtained, for instance, in fragmented scapula. The present study showed that MB was $90.9 \%$ accurate in both original sample and cross-validated sample, compared to ML, which showed less accuracy $(84.8 \%)$ in both original sample and cross-validated sample. These results were comparable with the study by Zhang et al. (2016), who showed $86.7 \%$ overall accuracy for MB and $84.8 \%$ overall accuracy for ML. In contrast, Torimitsu et al. (2016) and Papaioannou et al. (2012) stated a higher accuracy for ML compared to that with MB. The discrepancies between these studies may be attributed to variations in the structure of the scapula in different populations [10]. It may be also due to the different techniques used in the scapula measurements contributing to value discrepancies. For instance, in the study by Zhang et al. (2016), the anterior part of the scapular was used to measure MB compared to that by Torimitsu et al. (2016) and Papaioannou et al. (2012), who used posterior view of scapula for measurements.

Evidently, the overall accuracy of the equation was higher (92.4\%) when both $M B$ and $M L$ were used in combination, compared to using only a single parameter. Previous studies had stated that using multiple parameters; the accuracy can rise to $95 \%$ compared to using only a single parameter $[1,10,24]$. In addition, the Wilk's lambda had yielded good discrimination in the equation models. The Wilk's lambda for a single parameter was lower for $M L(0.41)$ than for $M B(0.43)$. In both parameters, the Wilk's Lambda showed a better separation in the equations. The results had indicated that by combining both parameters, the equation yielded good accuracy and reliability.

In the recent decades, CT scapula had frequently been used for sex estimation in several populations $[10,15,17,18]$. The main concern was to know whether utilization of CT was comparable with that using direct measurement on dry scapula bone. Few comparable studies were done to examine the reliability of the above methods. Stull et al. (2014) stated that CT scan is an acceptable option for the anthropologists, as the difference between CT image and true bone was only within the range of $\pm 2 \mathrm{~mm}$, which can be considered a close approximation [28]. In addition, Ali et al. (2018) had compared between dry bone and CT scan and documented that CT scan is a reliable method and has rapidly been used for human identification, especially in mass disaster. The application of CT scan is considerably useful and reliable, as an option for sex estimation, especially when there are only limited dry bone collections in many populations.

In this study, interobserver and intraobserver variability was analyzed to measure the reliability and repeatability of the scapula measurements. It is a pivotal matter in ensuring that the data collected were accurate and reproducible. The technical error of measurement is one of the accuracy indexes that have been adopted by the International Society Standardization Advancement in Kinanthropometry [24]. It can demonstrate the degree of accuracy in the measurements of parameters among observers. Intraobserver and interobserver variability in the currentstudy had shown acceptable rTEMvalues for intraobserver (1.5\%) and interobserver (2.0\%). In fact, the $R$ value in this study had exceeded the acceptable levels of $R$ that ranged between 0.97 and 0.99 [29].

\section{CONCLUSION}

This study had demonstrated the presence of sexual dimorphism in scapula, which could be used as an alternative for the identification of skeletonized remains in the Malaysian population. The discriminant function equations generated in this study can be useful in estimating sex. However, additional parameters for scapula in an extended sample are recommended to validate the findings in this study.

\section{ACKNOWLEGDMENTS}

Special thanks to Radiology Department, UKM for having access to the cases under study. The authors are grateful to UKM for the funding of this research project (Code Project No: FF-2018-37). The authors also wish to thank Mr Kamil Khalid for his contribution.

\section{AUTHORS' CONTRIBUTIONS}

Dr. Normaliza Omar, Dr. Siti Hanum Mohd Ali, Dr. Mohamed Swarhib Shafie, Dr. Nik Azuan Nik Ismail, Dr. Helmi Hadi, and Dr. Faridah Mohd Nor had contributed to the concept and design of the study, collection, analysis, and interpretation of data. Dr. Normaliza Omar and Dr. Faridah Mohd Nor had drafted and revised the article critically for the intellectual content, and final approval of the final version to be published.

\section{CONFLICTS OF INTEREST}

There are no conflicts of interest.

\section{REFERENCES}

1. Ozer I, Katayama K, Sağir M, Güleç E. Sex determination using the scapula in medieval skeletons from East Anatolia. Coll Antropol 2006;30:415-9.

2. Ali Z, Cox C, Stock MK, van Rilland EE, Rubio A, Fowler DR, et al. Estimating sex using metric analysis of the scapula by postmortem computed tomography. J Forensic Sci 2018;63:1346-9.

3. Krishan K, Chatterjee PM, Kanchan T, Kaur S, Baryah N, Singh RK, et al. A review of sex estimation techniques during examination of skeletal remains in forensic anthropology casework. Forensic Sci Int 2016;261:165.e1-8.

4. Syngle T, Kaur S, Garg N. Osteoporotic fracture risk in rheumatoid arthritis. Int J Pharm Sci 2018;10:106-9.

5. Steyn M, Işcan MY. Metric sex determination from the pelvis in modern greeks. Forensic Sci Int 2008;179:86.e1-6.

6. Steyn M, Işcan MY. Sex determination from the femur and tibia in South African whites. Forensic Sci Int 1997;90:111-9.

7. Kanz F, Fitzl C, Vlcek A, Frommlet F. Sex estimation using the femur of Austrians born in the $19^{\text {th }}$ to the middle of the $20^{\text {th }}$ century. Anthropol Anz 2015;72:117-27

8. Case DT, Ross AH. Sex determination from hand and foot bone lengths. J Forensic Sci 2007;52:264-70.

9. Maclaughlin SM, Oldale NM. Vertebral body diameters and sex prediction. Ann Hum Biol 1992;19:285-92.

10. Torimitsu S, Makino Y, Saitoh H, Sakuma A, Ishii N, Yajima D, et al. Sex estimation based on scapula analysis in a Japanese population using multidetector computed tomography. Forensic Sci Int 2016;262:285.e1-5.

11. El Morsi DA, Gaballah G, Mahmoud W, Tawfik AI. Sex determination in Egyptian population from scapula by computed tomography. J Forensic Res 2017;08:8-11.

12. Giurazza F, Schena E, Del Vescovo R, Cazzato RL, Mortato L, Saccomandi $\mathrm{P}$, et al. Sex determination from scapular length measurements by CT scans images in a Caucasian population. Conf Proc IEEE Eng Med Biol Soc 2013;2013:1632-5.

13. Papaioannou VA, Kranioti EF, Joveneaux P, Nathena D, Michalodimitrakis M. Sexual dimorphism of the scapula and the clavicle in a contemporary Greek population: Applications in forensic identification. Forensic Sci Int 2012;217:231.e1-7.

14. Peckmann TR, Scott S, Meek S, Mahakkanukrauh P. Sex estimation from the scapula in a contemporary Thai population: Applications for forensic anthropology. Sci Justice 2017;57:270-5.

15. Paulis MG, Samra MF. Estimation of sex from scapular measurements using chest CT in Egyptian population sample. J Forensic Radiol Imaging 2015;3:153-7.

16. Patriquin ML, Steyn M, Loth SR. Metric analysis of sex differences in South African black and white pelves. Forensic Sci Int 2005;147:119-27.

17. Zhang K, Cui JH, Luo YZ, Fan F, Yang M, Li XH, et al. Estimation of stature and sex from scapular measurements by three-dimensional volume-rendering technique using in Chinese. Leg Med (Tokyo) 2016;21:58-63.

18. Dine MM, Hassan HM. Ontogenetic study of the scapula among some Egyptians: Forensic implications in age and sex estimation using multidetector computed tomography. Egypt J Forensic Sci 2016;6:56-77.

19. Ibrahim A, Alias A, Nor FM, Swarhib M, Abu Bakar SN, Das S, et al. Study of sexual dimorphism of Malaysian crania: An important step in identification of the skeletal remains. Anat Cell Biol 2017;50:86-92.

20. Ibrahim A, Alias A, Nor FM, Swarhib M, Das S. Osteometric estimation 
of sex from mastoid triangle in Malaysian population. Asian J Pharm Clin Res 2018;11:303-7.

21. Alias A, Ibrahim A, Bakar SN, Rokhani F, Swarhib M, Nor FM. Morphometric analysis of coronoid process of mandible by $\mathrm{CT}$ in the Malaysian population : An important step for determination of sex. J Dent Sci Res Ther 2018;5:1-8.

22. Varghese AP, Philip B, Krishnakumar M, Kuriakose F, Rodrigues PA. Assessment of osteoporosis and anaemia risk in patients on anticonvulsant therapy. Int J Pharm Sci 2016;8:404-8.

23. Jamaiyah H, Geeta A, Safiza MN, Khor GL, Wong NF, Kee CC, et al. Reliability, technical error of measurements and validity of length and weight measurements for children under two years old in Malaysia. Med J Malaysia 2010;65 Suppl A:131-7.

24. Perini TA, de Oliveira GL, Ornelia JS, de Oliveira FP. Technical error of measurement in anthropometry. Rev Bras Med Esporte 2005;11:81-5.

25. Dabbs GR, Moore-Jansen PH. A method for estimating sex using metric analysis of the scapula. J Forensic Sci 2010;55:149-52.

26. Hudson A, Peckmann TR, Logar CJ, Meek S. Sex determination in a contemporary Mexican population using the scapula. J Forensic Leg Med 2016;37:91-6.

27. Koukiasa AE, Eliopoulos C, Manolis SK. Biometric sex estimation using the scapula and clavicle in a modern Greek population. Anthropol Anz 2017;74:241-6.

28. Stull KE, Tise ML, Ali Z, Fowler DR. Accuracy and reliability of measurements obtained from computed tomography 3D volume rendered images. Forensic Sci Int 2014;238:133-40.

29. Ulijaszek SJ, Kerr DA. Anthropometric measurement error and the assessment of nutritional status. Br J Nutr 1999;82:165-77. 